\title{
Treatment of community-acquired pneumonia
}

\author{
Donald E. Low MD
}

$\infty$ See related research paper by Vardakas and colleagues, page 1269

I $\mathrm{n}$ this issue of CMAJ, Vardakas and colleagues ${ }^{1}$ present the findings of a meta-analysis of treatment strategies for community-acquired pneumonia. Because pneumonia is a leading cause of death in the developed world, determining which antibiotics are most effective under which circumstances is critical. For treatment of mild to moderate community-acquired pneumonia, most regimens that include $\beta$-lactams, macrolides or fluoroquinolones provide similar rates of clinical success and survival. However, which regimen is superior for the treatment more severe disease that requires hospital admission remains controversial.

Most societies recommend combination therapy that includes a macrolide for treatment of severe communityacquired pneumonia., ${ }^{2,3}$ These recommendations are based mostly on large retrospective studies. It is not known whether the benefit seen with the addition of a macrolide to a $\beta$-lactam-based treatment is associated with the pathogens covered (including "atypical" pneumonia organisms such as Mycoplasma pneumoniae, Chlamydophila pneumoniae, and Legionella spp.) or with an anti-inflammatory effect specific to the macrolide class of antibiotics. A recent retrospective observational study of community-acquired pneumonia, based on data obtained from drug trials involving 4337 patients in 21 countries, reported that the pathogen, in cases where it could be isolated, was an atypical organism in $22 \%$ of cases. ${ }^{4}$ However, prospective studies have failed to provide evidence that the use of antimicrobials with activity against atypical pathogens improves outcomes. ${ }^{5}$

Long-term treatment with macrolide antibiotics such as clarithromycin, erythromycin, or azithromycin can decrease production of inflammatory cytokines from airway cells. Such treatment is routinely used for cystic fibrosis and diffuse panbronchiolitis; however, the mechanisms of action of these drugs have not been fully elucidated. ${ }^{6}$ Whether the favourable outcome seen with short-term treatment with macrolides in community-acquired pneumonia is a result of an anti-inflammatory effect is unknown. The efficacy of respiratory fluoroquinolones alone or as an alternative to newer generation macrolides in combination therapy in patients with severe community-acquired pneumonia has not been prospectively evaluated.

What can we learn from the meta-analysis by Vardakas and colleagues? Of the 1355 studies identified by the authors, only 23 studies that compared fluoroquinolones to $\beta$-lactams or macrolides (alone or in combination) met the criteria for inclusion. As in other meta-analyses, there were markedly different criteria among the studies for site of care (inpatient v. outpatient), route of antibiotic administration (intravenous v. oral), severity of illness and outcome measures. The high effectiveness of both fluoroquinolones and comparator antibiotic regimens is not surprising because most of the included

\section{Key points}

- Fluoroquinolones, macrolides and $\beta$-lactams are effective for the treatment of community-acquired pneumonia.

- Despite limited evidence, experts recommend that patients with community-acquired pneumonia be given at least 1 antimicrobial to treat atypical pathogens.

- Severe community-acquired pneumonia should be treated with a respiratory fluoroquinolone or a combination of macrolides and $\beta$-lactams.

- Prior exposure to a specific antimicrobial in the past 3 months or resistance exceeding $25 \%$ should lead clinicians to consider other alternatives.

patients had mild to moderate pneumonia. However, the authors found a significantly greater benefit of fluoroquinolone treatment in some subgroups of patients, including those who required admission to hospital or intravenous therapy. Interestingly, in the 8 randomized controlled trials that were blinded, no differences were seen in any of the outcome measures between the antibiotic regimens.

Details about patterns of antibiotic resistance were reported in only 13 trials. Resistance ranged from $8 \%-33 \%$ for macrolides and $0 \%-11.5 \%$ for $\beta$-lactams. Only one Staphylococcus aureus isolate resistant to levofloxacin was reported. Vardakas and colleagues suggest that the almost zero baseline resistance of the isolated pathogens to fluoroquinolones, in contrast to the higher resistance found for $\beta$-lactams and especially macrolides, may be an explanation for the higher treatment success observed with fluoroquinolones. However, whether the use of an antibiotic to which the infecting organism was resistant (discordant therapy) accounted for an increase in treatment failure could not be determined in this study. The burden of resistance was primarily due to pneumococcus, which accounted for most of the pathogens isolated.

The true impact of inadequate or discordant pneumococcal therapy has been difficult to determine. Studies of the effect of drug resistance on outcomes among patients with respiratory tract infections have focused on $\beta$-lactams in community-acquired pneumonia. In general, these studies have shown that outcomes are not affected by current levels of drug resistance; however, most of these studies had a small sample size and were unable control adequately for severity of illness and concordance of therapy. ${ }^{7}$ However, Daneman and colleagues ${ }^{8}$ showed, in a prospective population-based study, that treatment failures in pneumococcal bacteremia were significantly more common when the isolates were resistant to erythromycin than when they were susceptible. ${ }^{8}$

Dr. Low is with the Department of Microbiology, Mount Sinai Hospital, and the University Health Network, University of Toronto, Toronto, Ont. 
Strategies to minimize the risk of discordant therapy when treating community-acquired pneumonia include using a different class of antimicrobial drugs if the patient has taken that class in the previous 3 months or if resistance rates are in excess of $25 \%$. $^{9,10}$

The findings by Vardakas and colleagues support the current recommendations of the Infectious Diseases Society of America and the American Thoracic Society ${ }^{3}$ for the treatment of community-acquired pneumonia. These guidelines recommend a macrolide antibiotic or doxycycline for patients who were previously healthy and who have not used antimicrobial drugs within the previous 3 months. If comorbidities exist or if antimicrobials have been used in the previous 3 months, an alternative antimicrobial drug from a different class of antibiotics should be chosen. For patients admitted to hospital but not an intensive care unit, a respiratory fluoroquinolone or a $\beta$-lactam in combination with a macrolide is acceptable. Patients in an intensive care unit should be given a $\beta$-lactam and azithromycin or a respiratory fluoroquinolone. Following these recommendations will provide the best care for patients with community-acquired pneumonia.

Competing interests: None declared.

\section{REFERENCES}

1. Vardakas KZ, Siempos II, Grammatikos A, et al. Respiratory fluoroquinolones for the treatment of community-acquired pneumonia: a meta-analysis of randomized controlled trials. CMAJ 2008;179:1269-77.

2. British Thoracic Society of Standards of Care Committee (BTS). BTS guidelines for the management of community acquired pneumonia in adults. Thorax 2001; 56(Suppl 4):1-64.

3. Mandell LA, Wunderink RG, Anzueto A, et al. Infectious Diseases Society of America/American Thoracic Society consensus guidelines on the management of community-acquired pneumonia in adults. Clin Infect Dis 2007;44(Suppl 2): S27-72.

4. Arnold FW, Summersgill JT, Lajoie AS, et al. A worldwide perspective of atypical pathogens in community-acquired pneumonia. Am J Respir Crit Care Med 2007; 175:1086-93.

5. Oosterheert JJ, Bonten MJ, Hak E, et al. How good is the evidence for the recommended empirical antimicrobial treatment of patients hospitalized because of community-acquired pneumonia? A systematic review. J Antimicrob Chemother 2003; 52:555-63.

6. Oishi K, Sonoda F, Kobayashi S, et al. Role of interleukin-8 (IL-8) and an inhibitory effect of erythromycin on IL-8 release in the airways of patients with chronic airway diseases. Infect Immun 1994;62:4145-52.

7. Metlay JP, Singer DE. Outcomes in lower respiratory tract infections and the impact of antimicrobial drug resistance. Clin Microbiol Infect 2002;8(Suppl 2):1-11.

8. Daneman N, McGeer A, Green K, et al. Macrolide resistance in bacteremic pneumococcal disease: implications for patient management. Clin Infect Dis 2006;43:432-8

9. Daneman N, Low DE, McGeer A, et al. At the threshold: defining clinically meaningful resistance thresholds for antibiotic choice in community-acquired pneumonia. Clin Infect Dis 2008;46:1131-8.

10. Vanderkooi OG, Low DE, Green K, et al. Predicting antimicrobial resistance in invasive pneumococcal infections. Clin Infect Dis 2005;40:1288-97.

Correspondence to: Dr. Donald E. Low, Department of Microbiology, Mount Sinai Hospital, Rm. 1487, 600 University Ave., Toronto ON M5G 1X5; fax 416 586-8746;

dlow@mtsinai.on.ca
Every year, we distribute more than 1.5 million copies of CMAJ to Canadian physicians and health professionals around the world.

\section{CMAJ}

Medical knowledge that matters

Orders and information:

CMA Member Service Centre

tel 888 855-2555 or

$613731-8610 \times 2307$

fax $613236-8864$

cmamsc@cma.ca

Chaque année, nous distribuons de par le monde plus de 1,5 million d'exemplaires du JAMC aux médecins et aux professionnels de la santé.

\section{Le JAMC}

des connaissances médicales

qui comptent

Abonnements et demandes de renseignements :

Centre des services aux membres de l'AMC

tél 888 855-2555 ou

$613731-8610 \times 2307$

fax 613 236-8864

cmamsc@cma.ca

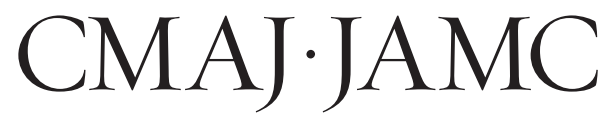

